\title{
Results of consecutive training procedures in pediatric cardiac surgery
}

Serban C Stoica ${ }^{1}$, David N Campbell ${ }^{2^{*}}$

\begin{abstract}
This report from a single institution describes the results of consecutive pediatric heart operations done by trainees under the supervision of a senior surgeon. The 3.1\% mortality seen in 1067 index operations is comparable across procedures and risk bands to risk-stratified results reported by the Society of Thoracic Surgeons. With appropriate mentorship, surgeons-in-training are able to achieve good results as first operators.
\end{abstract}

\section{Background}

Congenital heart surgery evolved from experimental lifesaving operations to treatment algorithms, risk stratification and quality control. This environment challenges the transfer of skills to new recruits. A variety of perceptions may hamper training: time or team constraints, procedure complexity, trainee's ability, trainer's commitment, lack of 'chemistry' between mentor and apprentice, patient's family demands or a combination of these. Many talented surgeons have learned 'by osmosis', through closely assisting an expert. If one gets better by performing rather than seeing a task, then regardless of aptitude it is preferable to progress from assistant to operator while still a trainee. To reduce the variability in exposure the newly developed certificate of congenital training in the US has strict requirements for the number and types of primary surgeon cases [1]. We report in this context the results of a pediatric attending (DNC) with special interest in training.

\section{Patients and Methods}

Whenever a trainee is available it has been the senior author's policy that he/she is the primary surgeon, remaining on the operator's side throughout the case. We do not have surgical practitioners. (Procedures done at a non-academic institution as well as congenital cases done at the adult university hospital are not reported here because of lacking risk stratification in these databases. Training however was the same. At the adult

\footnotetext{
* Correspondence: campbell.david@tchden.org

${ }^{2}$ Dept. of Pediatric Cardiac Surgery, Children's Hospital, Denver, Colorado, USA

Full list of author information is available at the end of the article
}

university hospital the practice consists of the full range of adult congenital disease and ductal ligations in the maternity, all of which became training cases for residents on service.) The current report therefore includes 1443 consecutive operations done under supervision by 7 fellows at Denver Children's Hospital between January 2003, when the Aristotle Basic Complexity score (ABCS) was introduced, and May 2009. In 33 cases where a trainee was not available another attending operated with the senior author assisting. Recently there was a change in referral patterns, the senior author taking responsibility for the Norwood program, and 6 stage I operations became 2 -attending procedures. These are the only nontraining cases in the series, leaving 1404 operations for analysis. To concentrate further on main procedures, after exclusion of chest reopening, delayed closure, pacemaker and patent ductus operations, wound and drainage procedures, but including chylothorax operations, 1067 index training cases were retained (Table 1). A comparison of their risk profile with that of the 33 nonNorwood 2-attending cases suggested no selection bias (ABCS, $7.1 \pm 2.0$ vs. $7.3 \pm 2.2, \mathrm{p}=0.60$, $\mathrm{t}$ test). 435 procedures $(40.7 \%)$ were in the levels 3 and 4 of complexity (ABCS $\geq 8.0$ ). The operative mortality for the 1067 index cases, defined by registry criteria [2], was $33(3.1 \%)$.

\section{Discussion}

Congenital training arrangements are summarized by Kogon's recent survey of 11 large programs, with 28 of 42 trainees responding (67\%) [1]. Encouragingly, the vast majority were satisfied with training overall however only 10 were satisfied with the operative experience. Each fellow performed a mean of 75 ( \pm 53 )
Ciomed Central

() 2010 Stoica and Campbell; licensee BioMed Central Ltd. This is an Open Access article distributed under the terms of the Creative Commons Attribution License (http://creativecommons.org/licenses/by/2.0), which permits unrestricted use, distribution, and reproduction in any medium, provided the original work is properly cited. 
Table 1 Patient details for 1067 index training cases

\begin{tabular}{|c|c|c|c|c|}
\hline \multicolumn{2}{|l|}{$\begin{array}{l}\text { Age (years), median } \\
\text { (interquartile range) } \\
\text { (range) }\end{array}$} & \multicolumn{3}{|c|}{$0.7(0.2,7.1)(0.0,44.1)$} \\
\hline $\begin{array}{l}\text { Weight }(\mathrm{kg}) \text {, median } \\
\text { (interquartile range) } \\
\text { (range) }\end{array}$ & & \multicolumn{3}{|c|}{$6.9(3.9,20.6)(0.9,178.2)$} \\
\hline $\begin{array}{l}\text { Basic Aristotle Score, } \\
\text { mean (standard } \\
\text { deviation) (range) }\end{array}$ & & \multicolumn{3}{|c|}{$7.1(2.0)(1.5,14.5)$} \\
\hline Procedure & $\mathrm{N}$ & $\begin{array}{l}\text { Hospital } \\
\text { mortality } \\
(\%)\end{array}$ & $\begin{array}{c}\text { Discharge \% } \\
\text { mortality STS } \\
\text { database [3] }\end{array}$ & $\begin{array}{c}\text { Late } \\
\text { mortality } \\
(\%)^{\mathrm{a}}\end{array}$ \\
\hline $\begin{array}{l}\text { Coarctation of the } \\
\text { aorta, arch surgery, } \\
\text { aortic aneurysm }\end{array}$ & 148 & $5(3.4)$ & N/a & 0 \\
\hline $\begin{array}{l}\text { Ventricular septal } \\
\text { defect (incl. } 1 \text { hybrid } \\
\text { perventricular) }\end{array}$ & 133 & 0 & $0-1.1$ & 0 \\
\hline Heart transplantation & 81 & $5(6.2)$ & 6.0 & $2(2.5)$ \\
\hline $\begin{array}{l}\text { ECMO cannulation/ } \\
\text { decannulation }\end{array}$ & 72 & $5(6.9)$ & $\mathrm{N} / \mathrm{a}$ & $4(5.5)$ \\
\hline $\begin{array}{l}\text { Right ventricular } \\
\text { outflow procedure }\end{array}$ & 69 & 0 & $4-5.8$ & 0 \\
\hline Atrio-ventricular canal & 57 & 0 & $1.3,4.5^{\mathrm{b}}$ & 0 \\
\hline Atrial septal defect & 39 & 0 & 1.4 & 0 \\
\hline $\begin{array}{l}\text { Tetralogy of Fallot } \\
\text { repair }\end{array}$ & 39 & $1(2.5)$ & $0.4-2.7$ & 0 \\
\hline $\begin{array}{l}\text { Systemic to pulmonary } \\
\text { shunt }\end{array}$ & 35 & $4(11.4)$ & 7.6 & $1(2.8)$ \\
\hline Glenn & 35 & 0 & 2 & 0 \\
\hline Vascular ring/sling & 29 & $1(3.4)$ & $\mathrm{N} / \mathrm{a}$ & 0 \\
\hline $\begin{array}{l}\text { Fontan (incl. } 2 \\
\text { conversions) }\end{array}$ & 27 & $1(3.7)$ & 3.9 & 0 \\
\hline Pericardial procedure & 27 & 0 & $\mathrm{~N} / \mathrm{a}$ & 0 \\
\hline $\begin{array}{l}\text { Ross, Konno, Ross- } \\
\text { Konno }\end{array}$ & 24 & $2(8.3)$ & $2.3^{c}$ & 0 \\
\hline $\begin{array}{l}\text { Mitral valve } \\
\text { replacement }\end{array}$ & 20 & $2(10)$ & $\mathrm{N} / \mathrm{a}$ & 0 \\
\hline $\begin{array}{l}\text { Pulmonary artery } \\
\text { banding debanding }\end{array}$ & 17 & 0 & $\mathrm{~N} / \mathrm{a}$ & 0 \\
\hline $\begin{array}{l}\text { Aortic stenosis sub-/ } \\
\text { supravalvar }\end{array}$ & 17 & 0 & $0^{d}$ & 0 \\
\hline $\begin{array}{l}\text { Partial anomalous } \\
\text { pulmonary venous } \\
\text { drainage } \\
\end{array}$ & 15 & 0 & N/a & 0 \\
\hline $\begin{array}{l}\text { Pleural drainage/ } \\
\text { decortication }\end{array}$ & 14 & 0 & $\mathrm{~N} / \mathrm{a}$ & 0 \\
\hline Pectus procedure & 13 & 0 & $\mathrm{~N} / \mathrm{a}$ & 0 \\
\hline $\begin{array}{l}\text { Total anomalous } \\
\text { pulmonary venous } \\
\text { drainage }\end{array}$ & 12 & $1(8.3)$ & 9.0 & 0 \\
\hline Diaphragm plication & 11 & 0 & $\mathrm{~N} / \mathrm{a}$ & 0 \\
\hline $\begin{array}{l}\text { Aortic root } \\
\text { replacement (incl. } 5 \\
\text { valve-sparing) }\end{array}$ & 11 & 0 & $\mathrm{~N} / \mathrm{a}$ & 0 \\
\hline $\begin{array}{l}\text { Aortic valve } \\
\text { replacement }\end{array}$ & 10 & 0 & N/a & 0 \\
\hline
\end{tabular}

Table 1 Patient details for 1067 index training cases (Continued)

\begin{tabular}{|c|c|c|c|c|}
\hline Truncus arteriosus & 8 & $2(25)$ & N/a & 0 \\
\hline $\begin{array}{l}\text { Tricuspid valve } \\
\text { procedure }\end{array}$ & 7 & 0 & N/a & 0 \\
\hline $\begin{array}{l}\text { Pulmonary artery } \\
\text { reconstruction }\end{array}$ & 7 & $1(14.3)$ & $\mathrm{N} / \mathrm{a}$ & 0 \\
\hline Coronary procedures & 7 & 0 & N/a & 0 \\
\hline PA-VSD procedure & 6 & 0 & $\mathrm{~N} / \mathrm{a}$ & 0 \\
\hline Mitral valve repair & 6 & $1(16.6)$ & 1.4 & 0 \\
\hline Norwood stage I & 6 & 0 & 31.4 & $1(16.6)$ \\
\hline $\begin{array}{l}\text { Pulmonary valve/Right } \\
\text { ventricular outflow } \\
\text { tract enlargement }\end{array}$ & 5 & 0 & $\mathrm{~N} / \mathrm{a}$ & 0 \\
\hline $\begin{array}{l}\text { Cor triatriatum, } \\
\text { supravalvar mitral ring }\end{array}$ & 4 & 0 & $\mathrm{~N} / \mathrm{a}$ & 0 \\
\hline $\begin{array}{l}\text { Double chambered } \\
\text { right ventricle }\end{array}$ & 4 & 0 & N/a & 0 \\
\hline $\begin{array}{l}\text { Ventricular assist } \\
\text { device (excl. } \\
\text { transplantation) }\end{array}$ & 3 & $1(33.3)$ & $\mathrm{N} / \mathrm{a}$ & 0 \\
\hline $\begin{array}{l}\text { Atrial septal defect } \\
\text { creation/enlargement }\end{array}$ & 3 & 0 & N/a & 0 \\
\hline Aortic valve repair & 3 & 0 & $\mathrm{~N} / \mathrm{a}$ & 0 \\
\hline Arterial switch & 2 & 0 & 2.0 & 0 \\
\hline Rastelli & 2 & 0 & $\mathrm{~N} / \mathrm{a}$ & 0 \\
\hline $\begin{array}{l}\text { Double outlet right } \\
\text { ventricle, } \\
\text { intraventricular tunnel }\end{array}$ & 2 & 0 & $\mathrm{~N} / \mathrm{a}$ & 0 \\
\hline $\begin{array}{l}\text { Aorto-pulmonary } \\
\text { window }\end{array}$ & 1 & 0 & $\mathrm{~N} / \mathrm{a}$ & 0 \\
\hline $\begin{array}{l}\text { Pulmonary vein } \\
\text { stenosis }\end{array}$ & 1 & 0 & $\mathrm{~N} / \mathrm{a}$ & 0 \\
\hline $\begin{array}{l}\text { One-and-a-half } \\
\text { ventricle repair }\end{array}$ & 1 & 0 & $\mathrm{~N} / \mathrm{a}$ & 0 \\
\hline Mustard & 1 & 0 & $\mathrm{~N} / \mathrm{a}$ & 0 \\
\hline Other & 33 & 0 & & 0 \\
\hline Total & 1067 & $33(3.1)$ & & $7(0.6)$ \\
\hline
\end{tabular}

$\mathrm{N} / \mathrm{a}$, not available; $\mathrm{a}$ - in addition to early mortality; $\mathrm{b}$ - for partial and complete AV canal respectively; c - for Ross operation; $d$ - for subvalvar aortic stenosis

operations and $51( \pm 42)$ open cases - note the variability. The majority did not perform any operations in the higher complexity range, as defined by a Risk Adjusted Congenital Heart Surgery Score of 4-6. The perception remains that apprenticeship, particularly for complex cases, continues even after training is over. We agree this is a reasonable expectation.

This report shows that the congenital operative experience can be maximized. All training deterrents enumerated in the introduction were consistently neutralized. By including consecutive patients and trainees selection bias is eliminated. Despite a significant number of complex cases the early outcomes were good, 
comparable with reports from the Society of Thoracic Surgeons [3] (Table 1). Our conclusion is limited by the absence of prospectively collected data to demonstrate that morbidity, but also cost and long-term results are not affected. However, another study in adults showed that training and non-training cardiac cases have similar long-term outcomes [4]. In summary, operative training is possible in consecutive congenital cases without increased risk to patients. We do not advocate a blanket adoption of this by other teams. It should be attempted only when everybody is comfortable and, above all, never at the patients' expense.

\section{Author details}

'Dept. of Pediatric Cardiac Surgery, Bristol Heart Institute and Children's Hospital, Bristol, UK. Dept. of Pediatric Cardiac Surgery, Children's Hospital, Denver, Colorado, USA.

\section{Authors' contributions}

SCS and DNC wrote the paper, DNC is the program director and supervised the training of residents as described. Both authors read and approved the final manuscript.

\section{Competing interests}

The authors declare that they have no competing interests.

Received: 6 May 2010 Accepted: 8 November 2010

Published: 8 November 2010

\section{References}

1. Kogon BE: The training of congenital heart surgeons. J Thorac CardiovasC Surg 2006, 132:1280-4.

2. Jacobs JP, Mavroudis C, Jacobs ML, Maruszewski B, Tchervenkov Cl, LacourGayet $F$, et al: What is operative mortality? Defining death in a surgical registry database: a report of the STS congenital database taskforce and the joint EACTS-STS congenital database committee. Ann Thorac Surg 2006, 81:1937-41.

3. Jacobs JP, Lacour-Gayet FG, Jacobs ML, Clarke DR, Tchervenkov Cl, Gaynor JW, et al: Initial application in the STS congenital database of complexity adjustment to evaluate surgical case mix and results. Ann Thorac Surg 2005, 79:1635-49.

4. Stoica SC, Kalavrouziotis D, Martin BJ, Buth KJ, Hirsch GM, Sullivan JA, et al: Long-term results of heart operations performed by surgeons in training. Circulation 2008, 118:S1-6.

doi:10.1186/1749-8090-5-105

Cite this article as: Stoica and Campbell: Results of consecutive training procedures in pediatric cardiac surgery. Journal of Cardiothoracic Surgery 2010 5:105.

\section{Submit your next manuscript to BioMed Central and take full advantage of:}

- Convenient online submission

- Thorough peer review

- No space constraints or color figure charges

- Immediate publication on acceptance

- Inclusion in PubMed, CAS, Scopus and Google Scholar

- Research which is freely available for redistribution

Submit your manuscript at www.biomedcentral.com/submit 